\title{
ggfortify: Unified Interface to Visualize Statistical Results of Popular R Packages
}

by Yuan Tang, Masaaki Horikoshi, and Wenxuan Li

\begin{abstract}
The ggfortify package provides a unified interface that enables users to use one line of code to visualize statistical results of many R packages using ggplot2 idioms. With the help of ggfortify, statisticians, data scientists, and researchers can avoid the sometimes repetitive work of using the ggplot2 syntax to achieve what they need.
\end{abstract}

\section{Background}

$\mathrm{R}$ users have many plotting options to choose from, such as base graphics, grid graphics, and lattice graphics (Sarkar, 2008). Each has their own unique customization and extensibility options. In recent years, ggplot2 has emerged as a popular choice for creating visualizations (Wickham, 2009) and provides a strong programming model based on a "grammar of graphics" which enables methodical production of virtually any kind of statistical chart. The ggplot2 package makes it possible to describe a wide range of graphics with succinct syntax and independent components and is based on an objectoriented model that also makes it modular and extensible. It has become a widely used framework for producing statistical graphics in $\mathrm{R}$.

The distinct syntax of ggplot2 makes it a definite paradigm shift from base and lattice graphics and presents a somewhat steep learning curve for those used to existing $\mathrm{R}$ charting idioms. Often times users only want to quickly visualize some statistical results from key R packages, especially those focusing on clustering and time series analysis. Many of these packages provide default base plot() visualizations for the data and models they generate. These components require transformation before using them in ggplot2 and each of those transformation steps must be replicated by others when they wish to produce similar charts in their analyses. Creating a central repository for common/popular transformations and default plotting idioms would reduce the amount of effort needed by all to create compelling, consistent and informative charts. To achieve this, we provide a unified ggplot2 plotting interface to many statistics and machine-learning packages and functions in order to help these users achieve reproducibility goals with minimal effort.

The ggfortify (Horikoshi and Tang, 2015) package has a very easy-to-use and uniform programming interface that enables users to use one line of code to visualize statistical results of many popular $\mathrm{R}$ packages using ggplot2 as a foundation. This helps statisticians, data scientists, and researchers avoid both repetitive work and the need to identify the correct ggplot2 syntax to achieve what they need. With ggfortify, users are able to generate beautiful visualizations of their statistical results produced by popular packages with minimal effort.

\section{Software architecture}

There are many ways to extend the functionality of ggplot2. One straightforward way is through the use of S3 generic functions ${ }^{1}$. Specifically, it is possible to provide custom functions for:

- autoplot(), which enables plotting a custom object with ggplot2, and

- fortify(), which enables converting a custom object to a tidy "data.frame"

The ggforitfy package uses this extensibility to provide default ggplot2 visualizations and data transformations.

To illustrate this, we consider the implementation for fortify.prcomp() and autoplot.pca_common() used as a basis of other PCA related implementations:

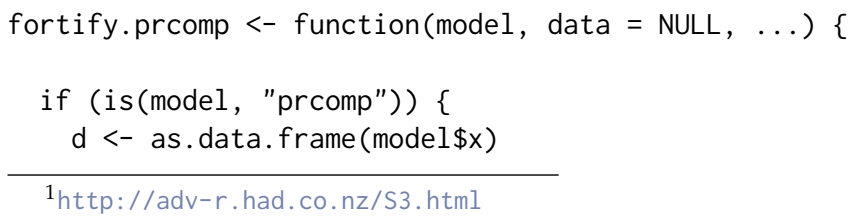

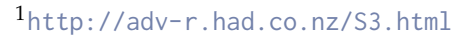




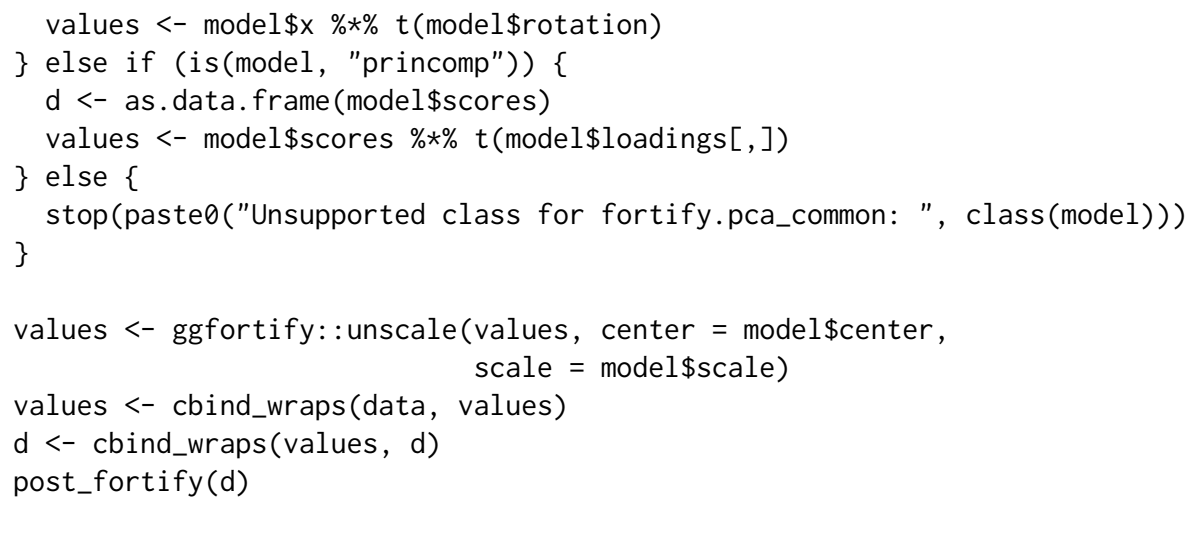

This S3 function recognizes "prcomp" objects and will extract the necessary components from them such as the matrix whose columns contain the eigenvectors in "rotation" and rotated data in " $x$ ", which can be drawn using autoplot() later on. The if() call is used here to handle different objects that are of essentially the same principal components family since they can be handled in the exactly same way once the necessary components are extracted from ggfortify.

The following autoplot.pca_common() function first calls fortify() to perform the component extraction for different PCA-related objects, then performs some common data preparation for those objects, and finally calls ggbiplot() internally to handle the actual plotting.

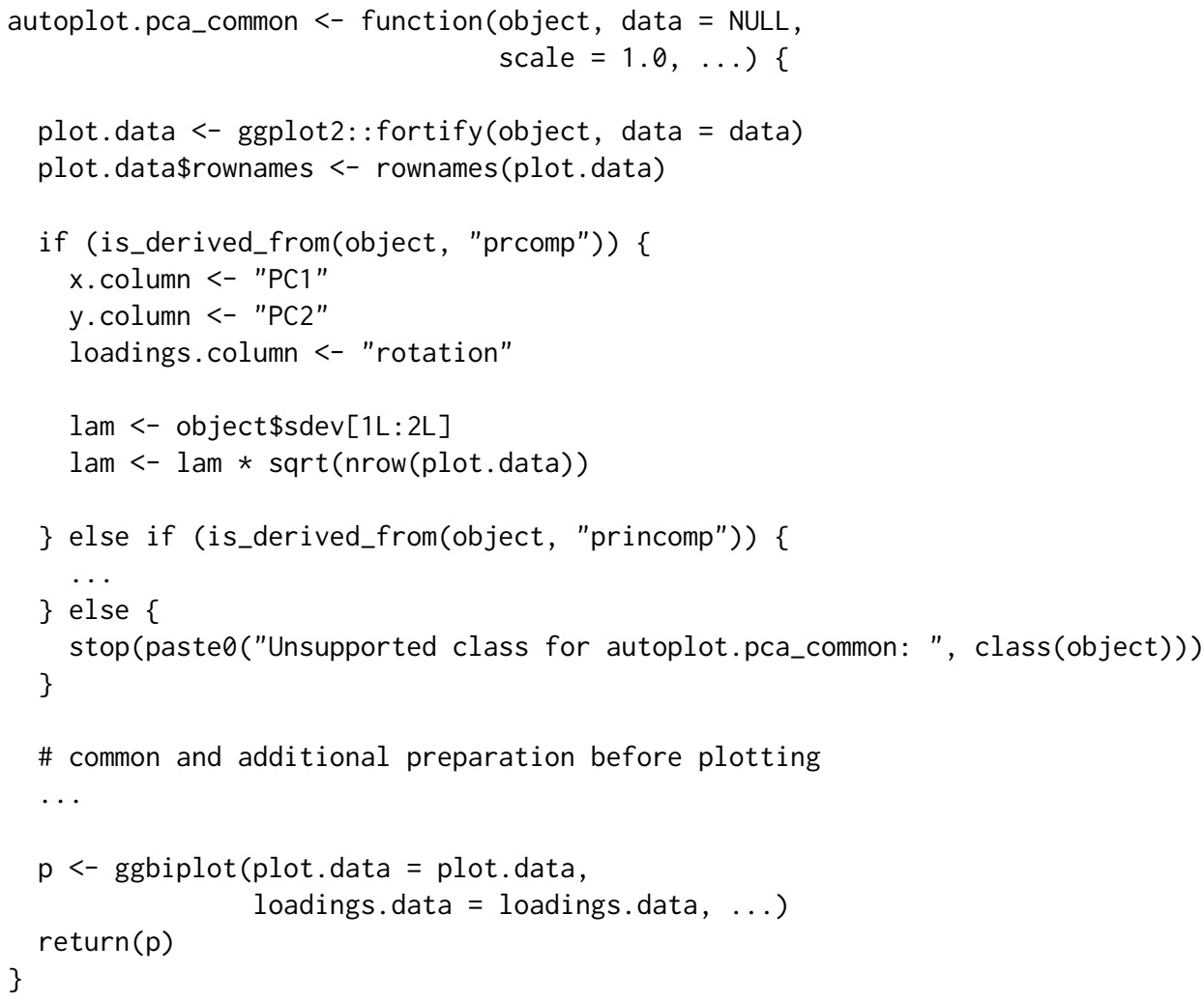

Once ggfortify is loaded, users have instant access to 38 pre-defined autoplot() functions and 36 pre-defined fortify() functions, enabling them to immediately autoplot() numerous types of objects or pass those objects directly to ggplot2 for manual customization. Furthermore, ggfortify is highly extensible and customizable and provides utility functions that make it easy for users to define autoplot() and fortify() methods for their own custom objects.

To present a streamlined API, ggfortify groups common implementations for various object-types, including:

- Time-series

- Principal components analysis (PCA), including clustering and multi-dimensional sacling (MDS) 
Table 1: Supported packages

\begin{tabular}{|c|c|c|c|}
\hline package & supported types & package & supported types \\
\hline base & "matrix", "table" & sp & $\begin{array}{l}\text { "SpatialPoints", } \\
\text { "SpatialPolygons", } \\
\text { "Line", "Lines", "Polygon", } \\
\text { "Polygons", "SpatialLines", } \\
\text { "SpatialLinesDataFrame", } \\
\text { "SpatialPointsDataFrame", } \\
\text { "SpatialPolygonsDataFrame" }\end{array}$ \\
\hline cluster & "clara", "fanny", "pam" & stats & $\begin{array}{l}\text { "Holtwinters", "lm", } \\
\text { "acf", "ar", "Arima", } \\
\text { "stepfun", "stl", "ts", } \\
\text { "cmdscale", "decomposed.ts", } \\
\text { "density", "factanal", "glm", } \\
\text { "kmeans", "princomp", "spec" }\end{array}$ \\
\hline changepoint & "cpt" & survival & "survfit","survvfit.cox" \\
\hline $\mathrm{dlm}$ & "dlmFilter", "dlmSmooth" & strucchange & $\begin{array}{l}\text { "breakpoints", } \\
\text { "breakpointsfull" }\end{array}$ \\
\hline fGarch & "fGARCH" & timeSeries & "timeSeries" \\
\hline forecast & $\begin{array}{l}\text { "bats", "forecast", "ets", } \\
\text { "nnetar" }\end{array}$ & tseries & "irts" \\
\hline fracdiff & "fracdiff" & vars & "varprd" \\
\hline glmnet & "cv.glmnet", "glmnet" & xts & "xts" \\
\hline KFAS & "KFS", "signal" & zoo & "zooreg" \\
\hline $\begin{array}{l}\text { lfda } \\
\text { maps }\end{array}$ & $\begin{array}{l}\text { "lfda", "klfda", "self" } \\
\text { "map" }\end{array}$ & MASS & "isoMDS", "sammon" \\
\hline
\end{tabular}

- $1 \mathrm{~d} / 2 \mathrm{~d}$ kernel density estimation (KDE)

- Survival analysis

- Cartography

A list of currently supported packages and classes can be found in Table 1. Additional packages that are in development are not shown here but more than 50 object types are supported by ggfortify. Feedback is being collected from users ${ }^{2}$ for possible bug fixes and future enhancements.

\section{Illustrations}

As previously stated, ggfortify provides methods that enable ggplot2 to work with objects in different classes from different $\mathrm{R}$ packages. The following subsections illustrate how to use ggfortify to plot results from several of these packages.

\section{Principal components analysis}

The ggfortify package defines both fortify() and autoplot() methods for the two core PCA functions in the stats package: stats: : prcomp() and stats: : princomp(). The values returned by either function can be passed directly to ggplot2: : autoplot() as illustrated in the following code and in Figure 1. Note that users can also specify a column to be used for the colour aesthetic.

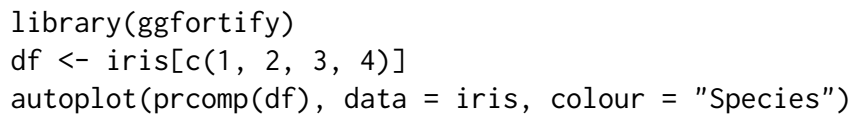

If label = TRUE is specified, as shown in Figure 2, ggfortify will draw labels for each data point. Users can also specify the size of the labels via label. size. If shape = FALSE is specified, the shape of the data points will be removed, leaving only the labels on the plot.

autoplot $(\operatorname{prcomp}(d f)$, data $=$ iris, colour = "Species", shape = FALSE, label.size $=3$ )

${ }^{2}$ https://github.com/sinhrks/ggfortify/issues 


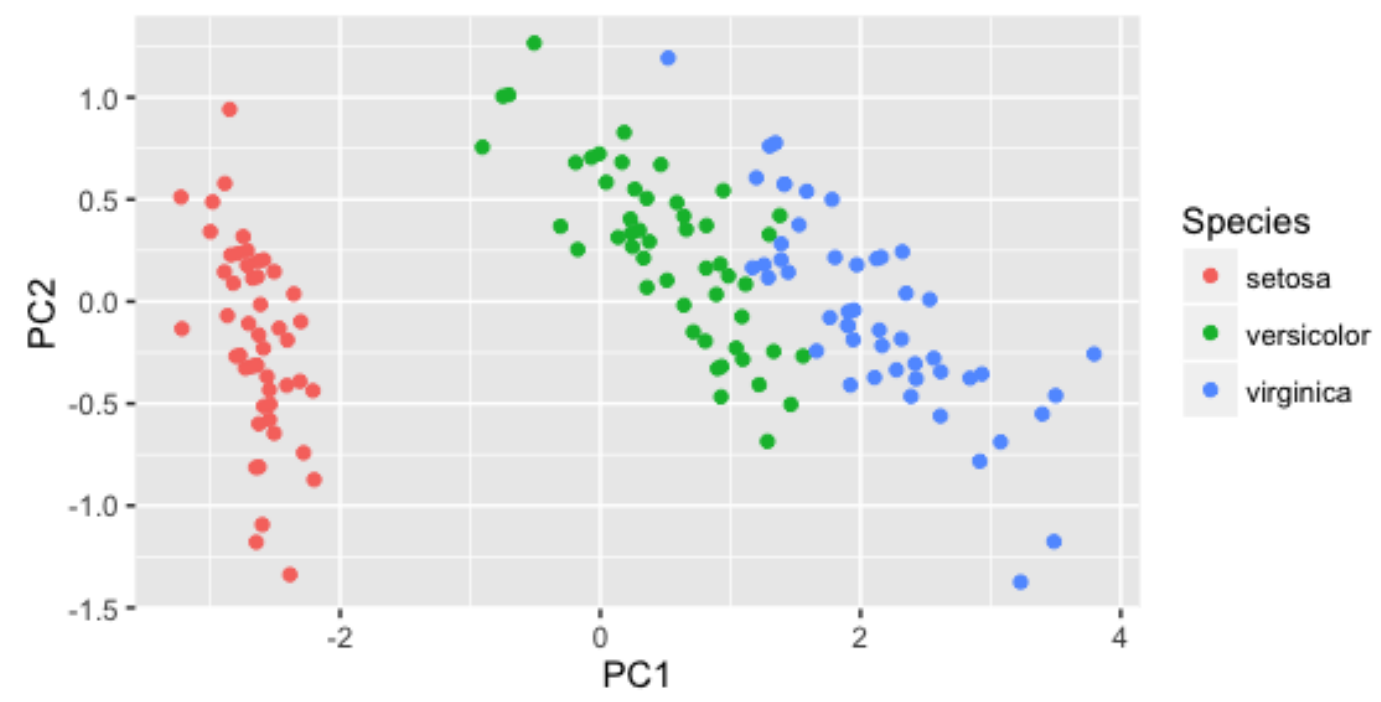

Figure 1: PCA with colors for each class.

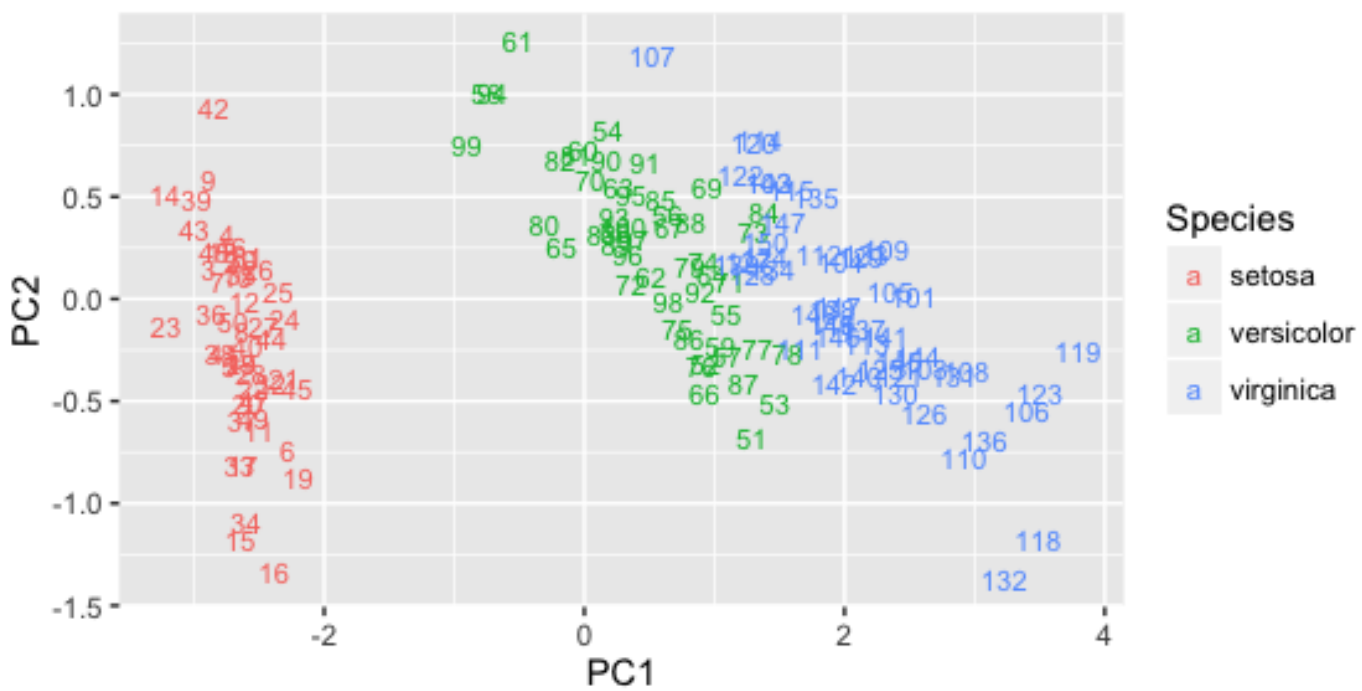

Figure 2: PCA with colors and labels for each class. 
The autoplot function returns the constructed ggplot2 object so users can apply additional ggplot2 code to further enhance the plot. For example:

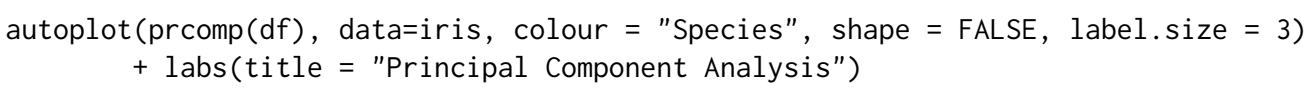

Users can also specify loadings = TRUE to draw the PCA eigen-vectors. More aesthetic options such as size and colors of the eigen-vector labels can also be specified as shown in Figure 3 and the following code:

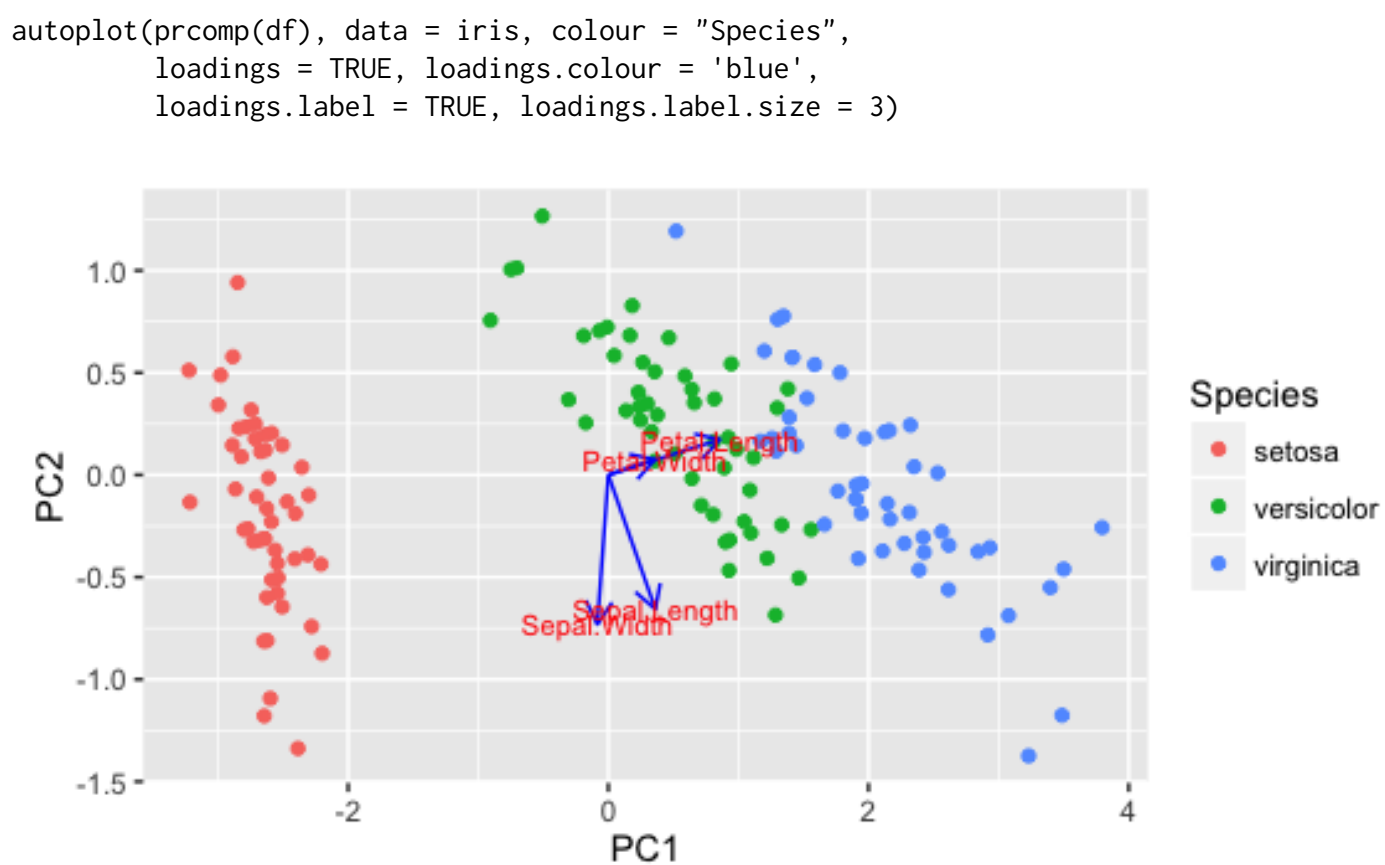

Figure 3: PCA with eigen-vectors and labels.

\section{Linear models}

The ggfortify function is able able to interpret $\operatorname{lm}($ ) fitted model objects and allows the user to select the subset of desired plots through the which parameter (just like the plot. $1 \mathrm{~m}()$ function). The ncol and nrow parameters also allow users to specify the number of subplot columns and rows, as seen in Figure 4 and the following code:

$\operatorname{par}(\mathrm{mfrow}=\mathrm{c}(1,2))$

$\mathrm{m}<-\operatorname{lm}$ (Petal. Width Petal.Length, data = iris)

autoplot $(\mathrm{m}$, which $=1: 6$, ncol $=3$, label. size $=3)$

Many plot aesthetics can be changed by using the appropriate named parameters. For example, the colour parameter is for coloring data points, the smooth. colour parameter is for coloring smoothing lines and the ad.colour parameter is for coloring the auxiliary lines, as demonstrated in Figure 5 and the following code:

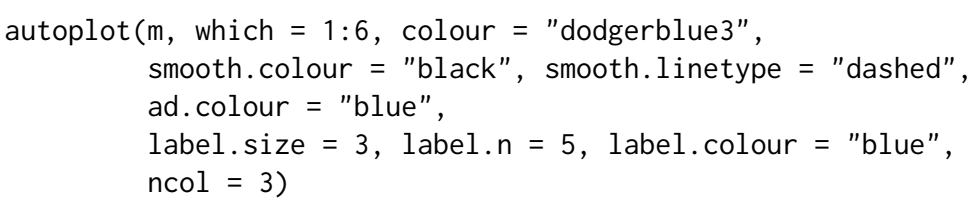

\section{Clustering}

The ggfortify package also supports various objects like "clara", "fanny", "pam", "kmeans", and "Ifda", from the cluster (Maechler et al., 2015) and lfda (Tang and Deane-Mayer, 2016) packages. It automatically infers the object type and plots the results from those packages using ggplot2 with a single function call. Users can specify frame = TRUE to easily draw the clustering boundaries as seen in Figure 6 and the following code: 

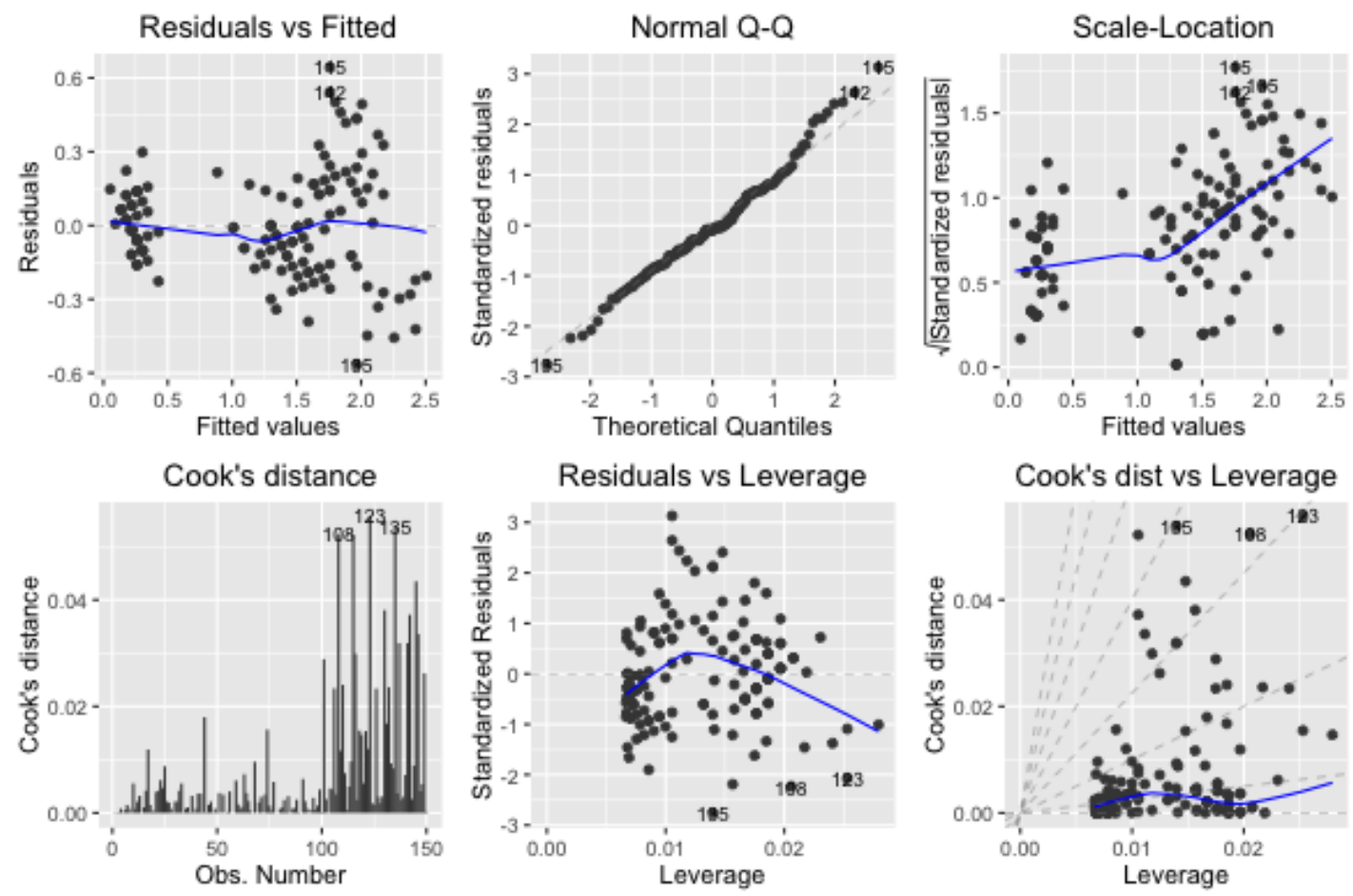

Figure 4: Linear model results.
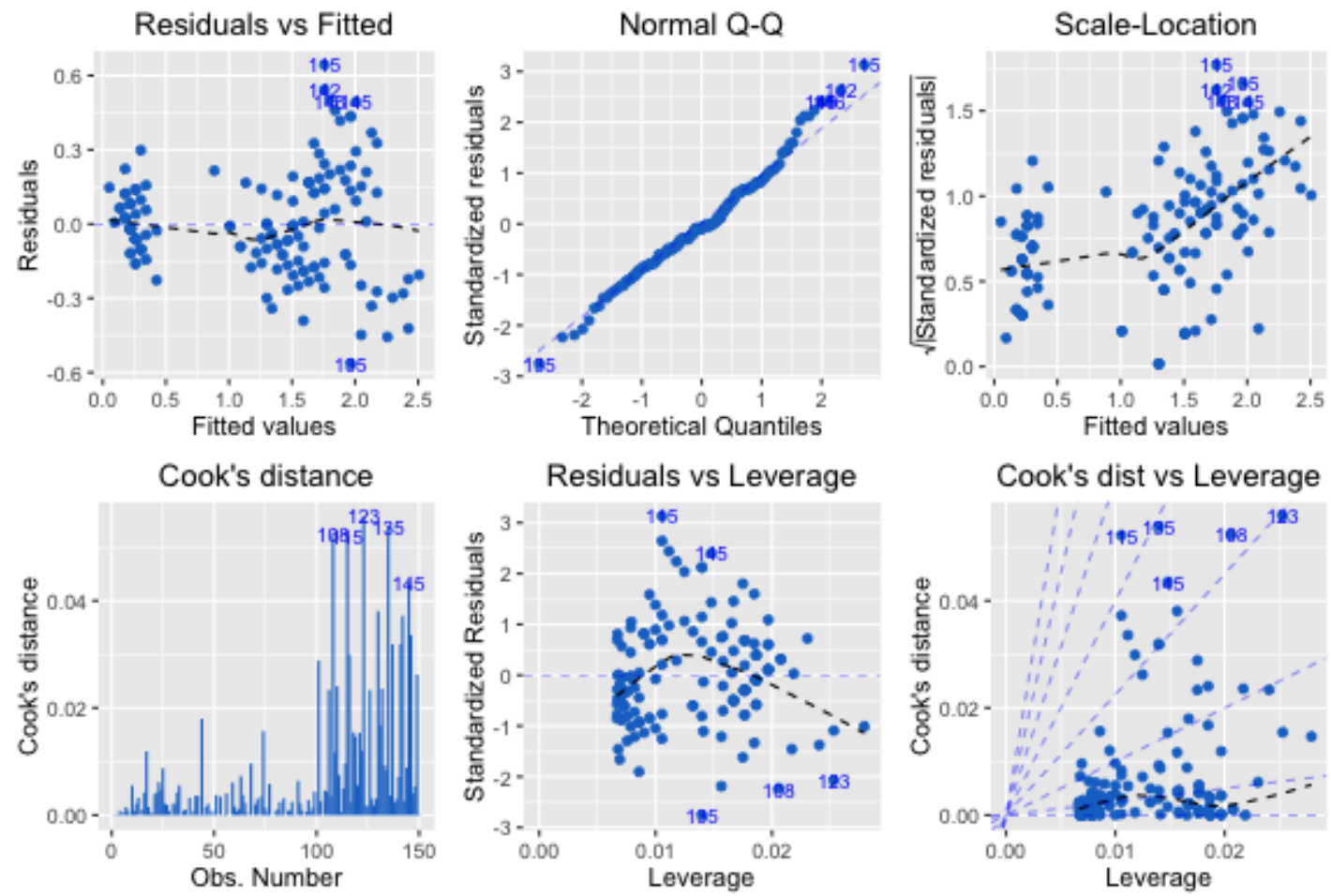

Figure 5: Linear model results with specified options. 
library (cluster)

autoplot (fanny (iris $[-5], 3)$, frame $=$ TRUE)

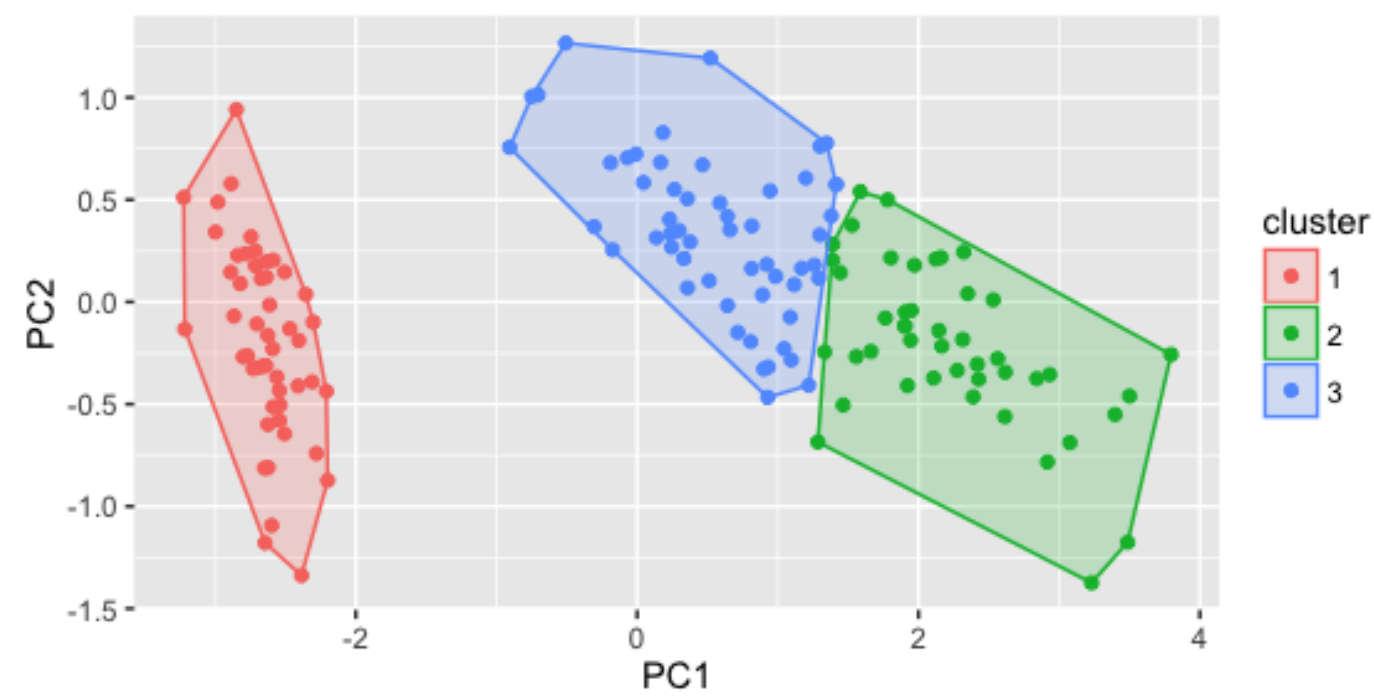

Figure 6: Clustering with boundaries.

As illustrated in Figure 7 with frame. type = "norm", by specifying frame. type users are able to draw boundaries of different shapes. The different frame types can be found in frame.type option in ggplot2: :stat_ellipse().

autoplot (pam(iris[-5], 3), frame = TRUE, frame.type = "norm")

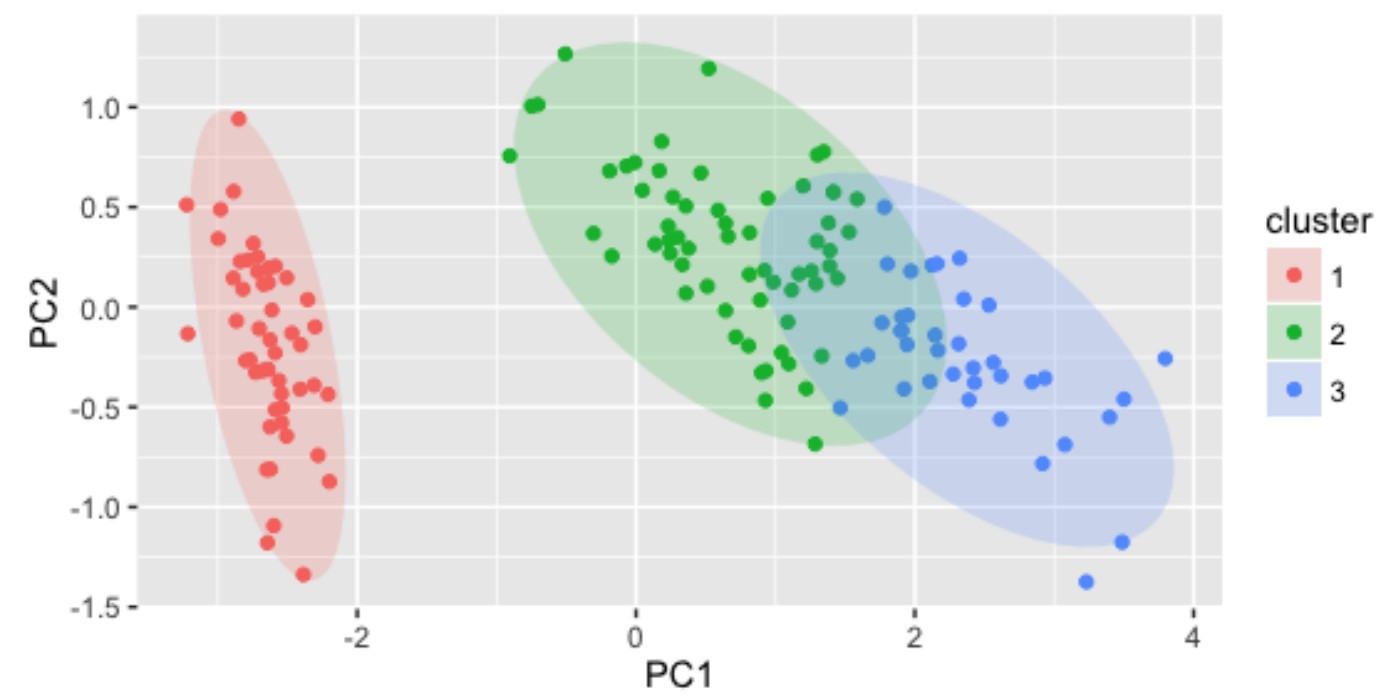

Figure 7: Clustering with boundaries in ellipse shape.

\section{Time series}

The ggfortify package makes it much easier to visualize time series objects using ggplot 2 and provides autoplot () and fortify() implementatons for ojects from many time series libraries such as zoo (Zeileis and Grothendieck, 2005), xts (Ryan and Ulrich, 2014), and timeSeries (Team et al., 2015).

Here is an example of using ggfortify to plot the AirPassengers example time series data set from the timeSeries package, specifying color via ts. colour, geometric shape via ts.geom as seen in Figure 8, Figure 9, and Figure 10: 
library(timeSeries)

autoplot(as.timeSeries(AirPassengers), ts.colour = "dodgerblue3")

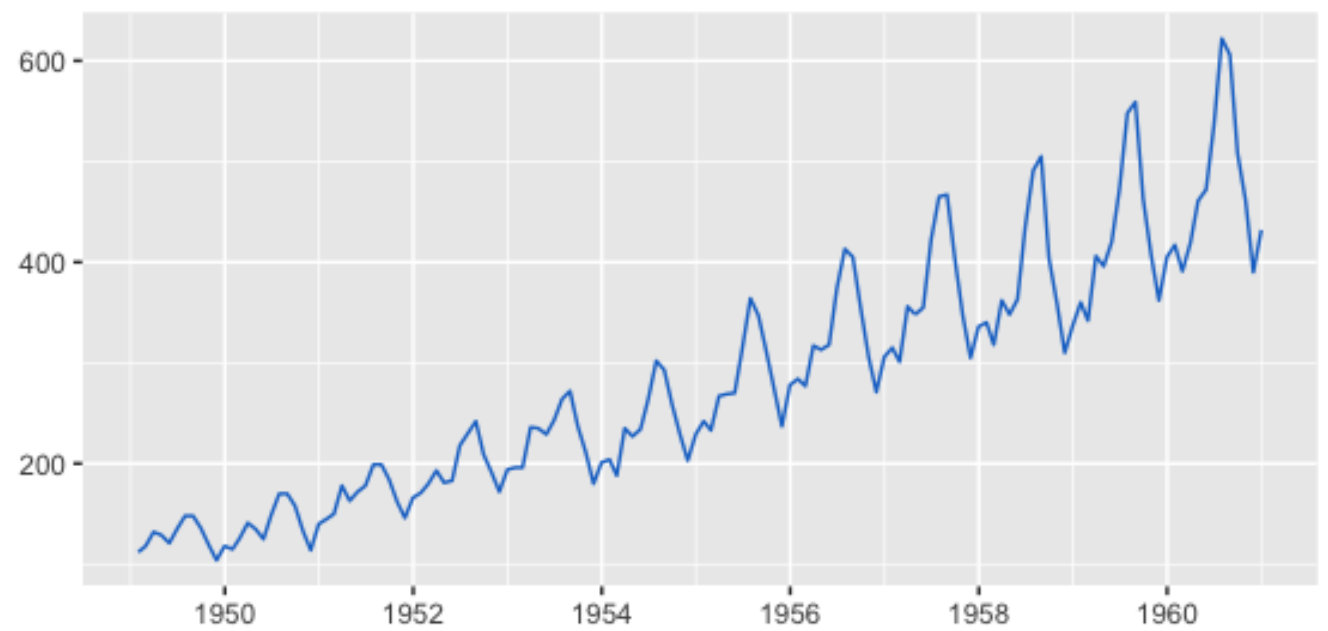

Figure 8: AirPassengers time series.

autoplot (AirPassengers, ts.geom = "bar", fill = "blue")

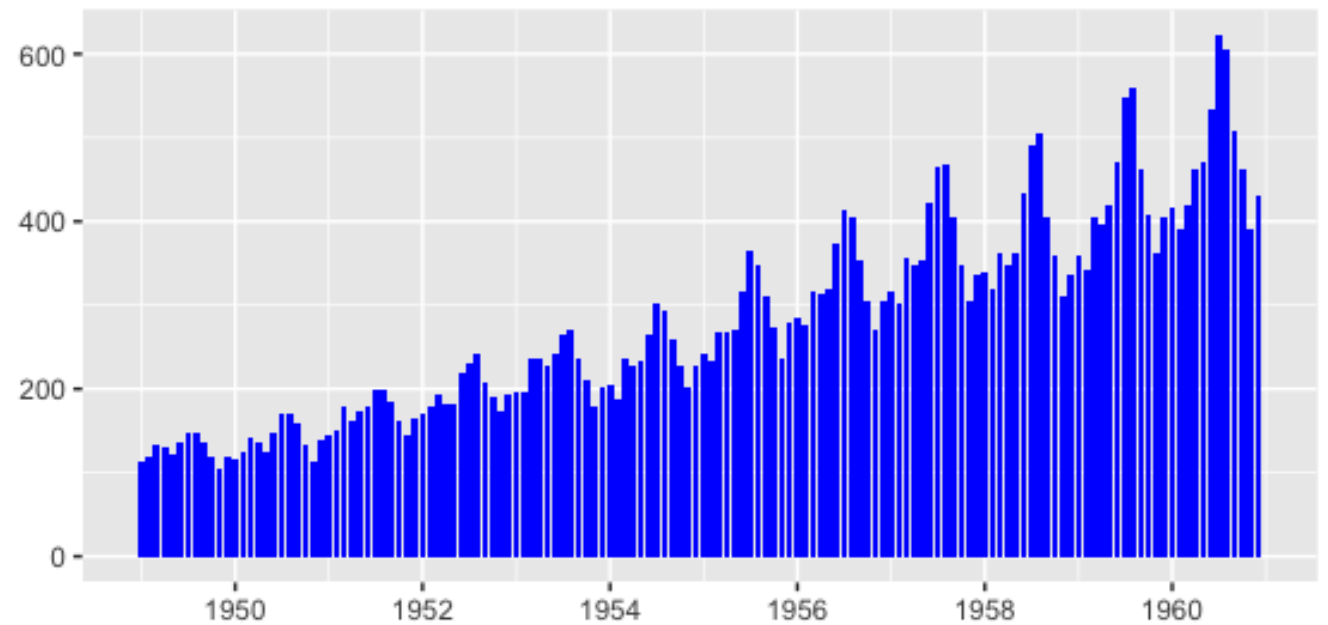

Figure 9: AirPassengers time series in bar shape.

autoplot (AirPassengers, ts.geom = "point", shape $=3$ )

\section{Forecasting}

Forecasting packages such as forecast (Hyndman, 2015), changepoint (Killick et al., 2016), strucchange (Zeileis et al., 2002), and dlm (Petris, 2010), are popular choices for statisticians and researchers. Predictions and statistical results from those packages can now be plotted automatically with ggplot2 using the functions provided by ggfortify. Note that in these cases the order of loading packages matters. For example, since forecast has its own autoplot() function, if it is loaded before ggfortify, the autoplot() function in forecast will be used instead.

The ggfortify function automatically plots the original and smoothed line from Kalman filter function in the dlm package as shown in Figure 11. 


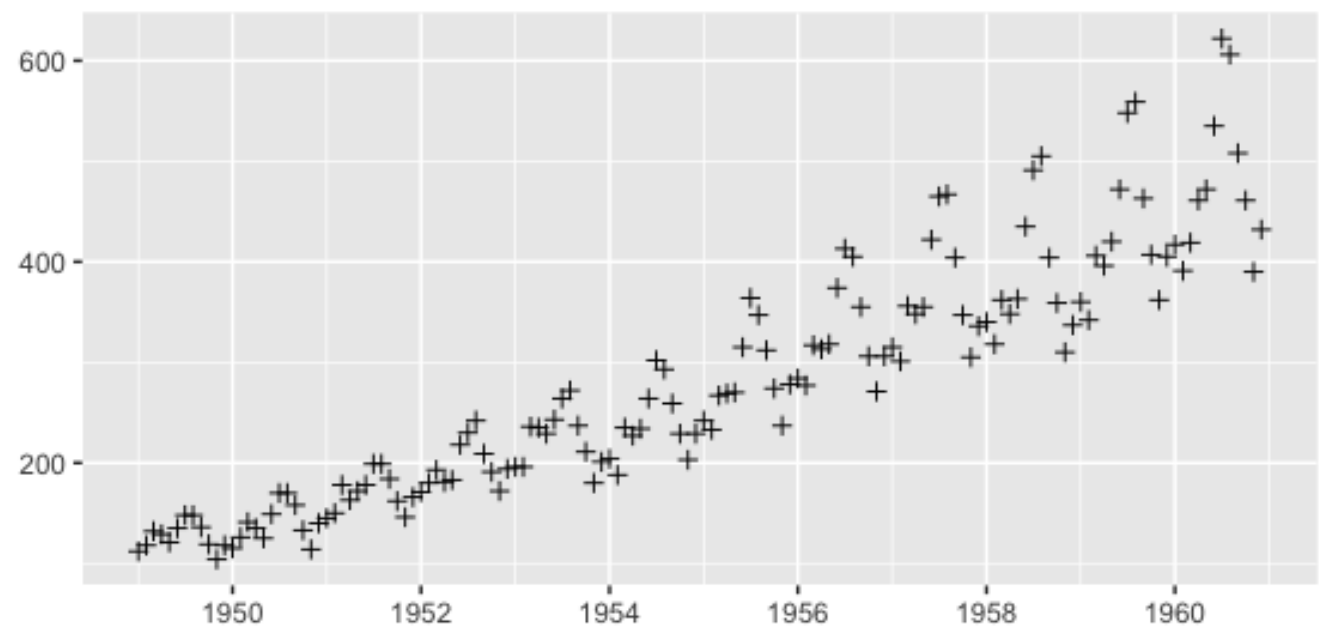

Figure 10: AirPassengers time series in point shape.
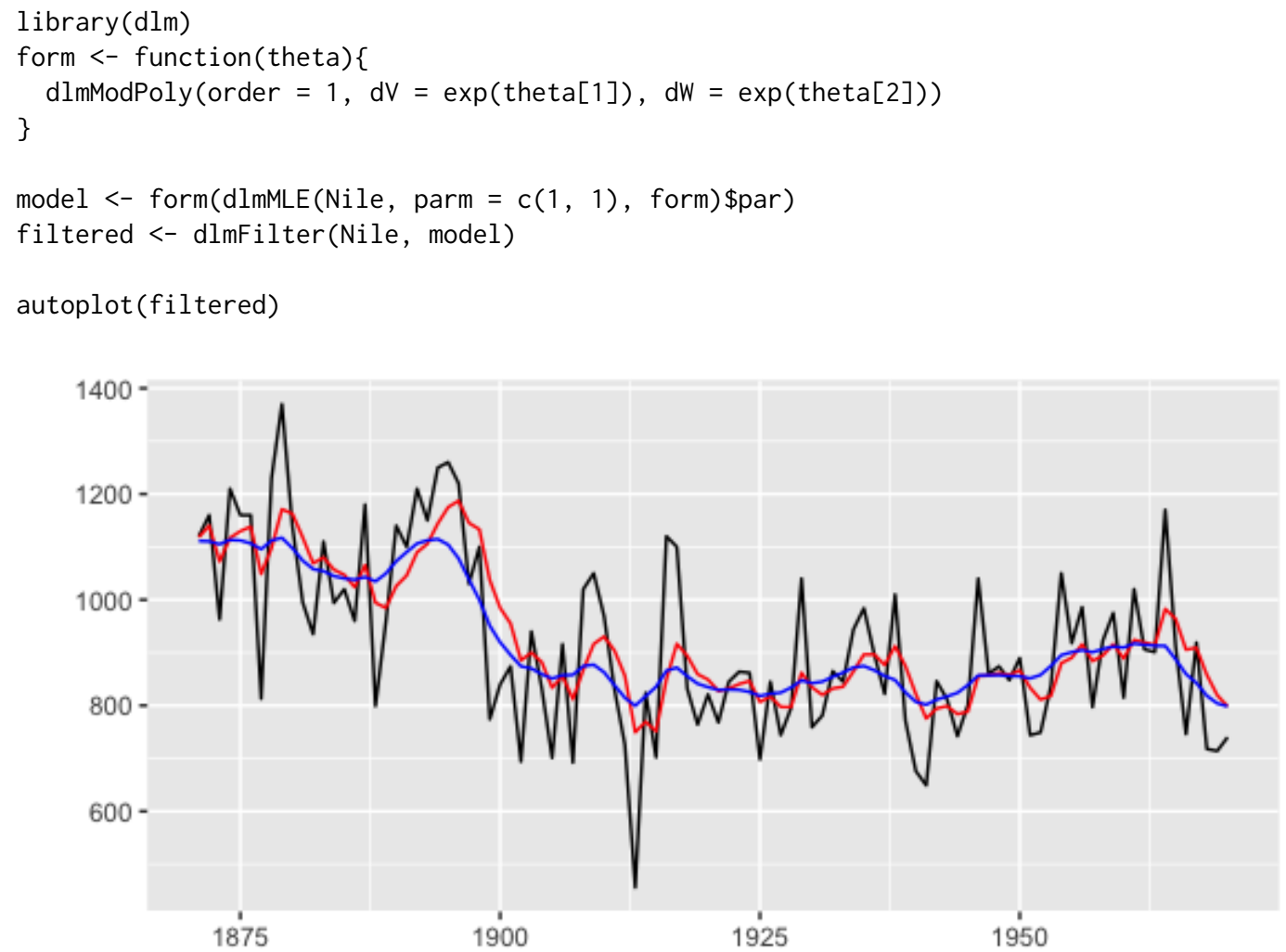

Figure 11: Smoothed time series by Kalman filter.

The ggfortify package automatically plots the change points with optimal positioning for the AirPassengers data set found in the changepoint package using the cpt.meanvar () function, shown in Figure 12 .

library (changepoint)

autoplot (cpt . meanvar (AirPassengers))

As well, ggfortify plots the optimal break points where possible structural changes happen in the regression models built by the strucchange: : breakpoints(), shown in Figure 13.

library (strucchange)

autoplot (breakpoints $(\mathrm{Nile} \sim 1)$, ts.colour $=$ "blue", ts.linetype $=$ "dashed", cpt. colour = "dodgerblue3", cpt.linetype = "solid") 


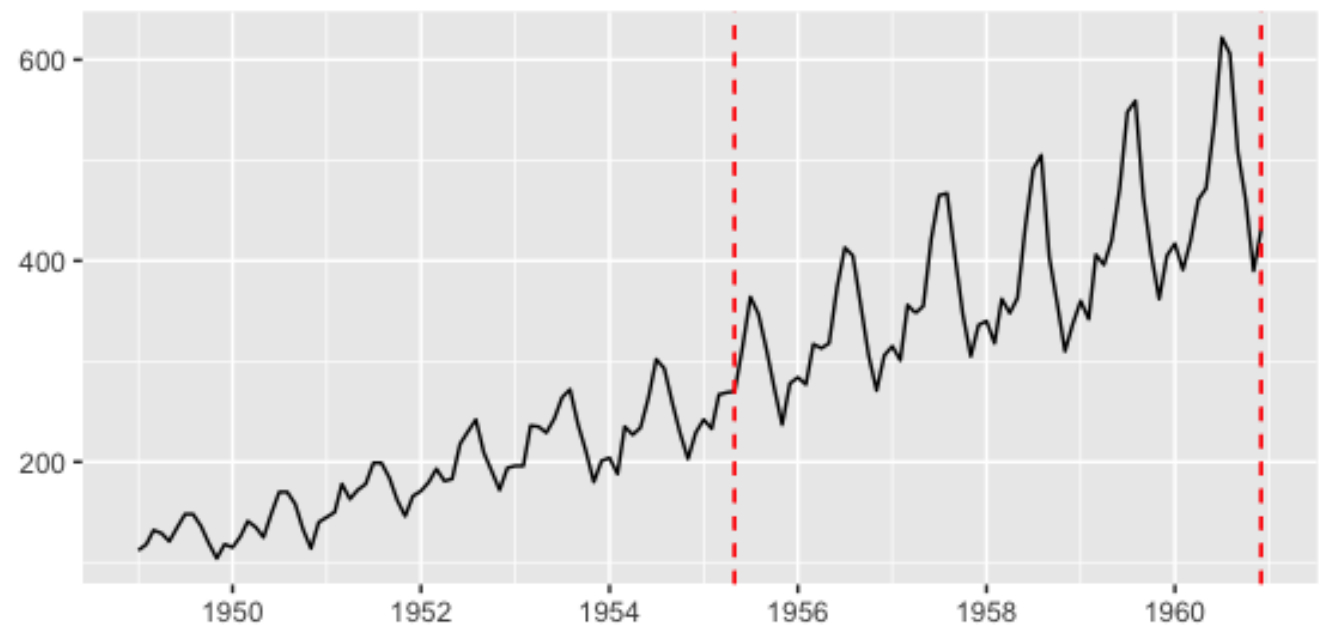

Figure 12: Change points with optimal positioning for AirPassengers.

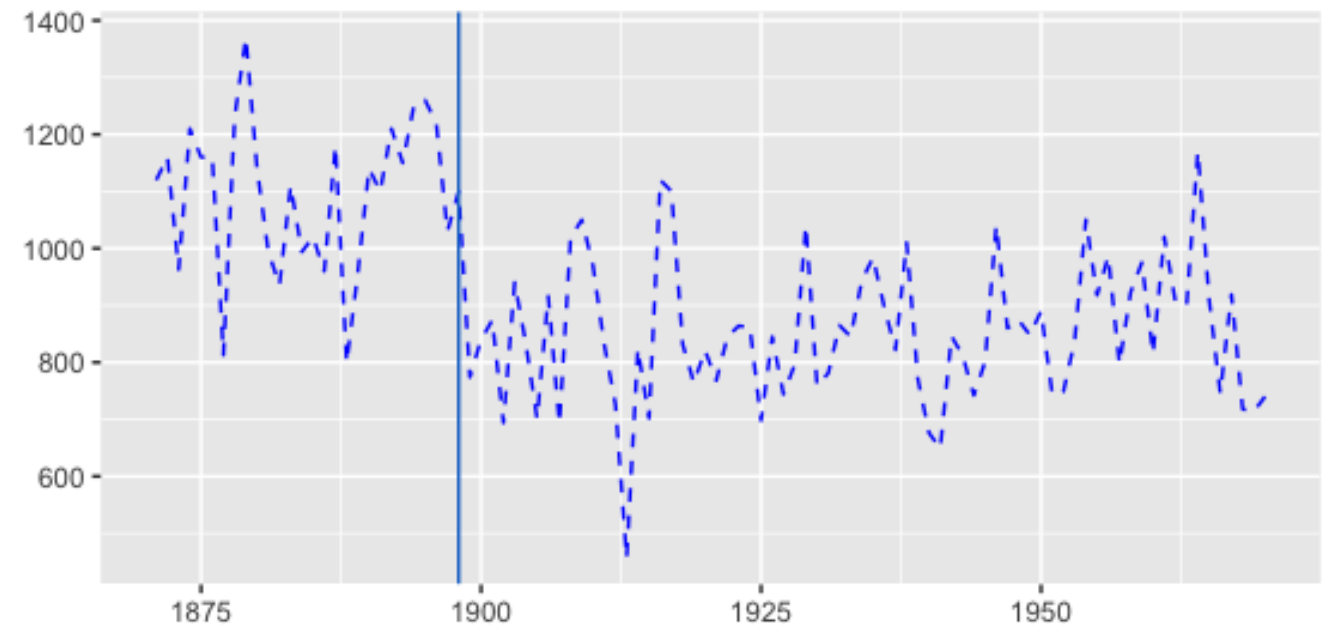

Figure 13: Optimal break points with possible structural changes.

\section{Future development}

We welcome suggestions and contributions from others. Providing default autoplot() and fortify() methods for additional $\mathrm{R}$ objects means researchers will spend less time focusing on ggplot2 plotting details and more time on their work and research. We are have provided a Github repository https: //github.com/sinhrks/ggfortify where users can test out development versions of the package and provide feature requests, feedback and bug reports. We encourage you to submit your issues and pull requests to help us make this package better for the $\mathrm{R}$ community.

\section{Summary}

The ggfortify package provides a very simple interface to streamline the process of plotting statistical results from many popular $R$ packages. Users can spend more time and focus on their analyses instead of figuring out the details of how to visualize their results in ggplot2. 


\section{Acknowledgement}

We sincerely thank all developers for their efforts behind the packages that ggfortify depend on, namely, dplyr (Wickham and Francois, 2015), tidyr (Wickham, 2016b), gridExtra (Auguie, 2016), and scales (Wickham, 2016a).

\section{Bibliography}

B. Auguie. gridExtra: Miscellaneous Functions for "Grid" Graphics, 2016. URL http://CRAN. R-project. org/package=gridExtra. R package version 2.2.1. [p484]

M. Horikoshi and Y. Tang. ggfortify: Data Visualization Tools for Statistical Analysis Results, 2015. URL http: //CRAN. R-project. org/package=ggfortify. R package version 0.1.0. [p474]

R. J. Hyndman. forecast: Forecasting functions for time series and linear models, 2015. URL http://github. com/robjhyndman/forecast. R package version 6.2. [p481]

R. Killick, K. Haynes, and I. A. Eckley. changepoint: An R package for changepoint analysis, 2016. URL http://CRAN.R-project.org/package=changepoint. R package version 2.2.1. [p481]

M. Maechler, P. Rousseeuw, A. Struyf, M. Hubert, and K. Hornik. cluster: Cluster Analysis Basics and Extensions, 2015. R package version 2.0.3 - For new features, see the 'Changelog' file (in the package source). [p478]

G. Petris. An R package for dynamic linear models. Journal of Statistical Software, 36(12):1-16, 2010. URL http: //www. jstatsoft.org/v36/i12/. [p481]

J. A. Ryan and J. M. Ulrich. xts: eXtensible Time Series, 2014. URL http://CRAN.R-project.org/ package=xts. R package version 0.9-7. [p480]

D. Sarkar. Lattice: Multivariate Data Visualization with R. Springer, New York, 2008. URL http: //Imdvr.r-forge.r-project.org. ISBN 978-0-387-75968-5. [p474]

Y. Tang and Z. Deane-Mayer. lfda: Local Fisher Discriminant Analysis, 2016. URL https: //github . com/ terrytangyuan/Ifda. R package version 1.1.1. [p478]

R. C. Team, D. Wuertz, T. Setz, and Y. Chalabi. timeSeries: Rmetrics - Financial Time Series Objects, 2015. URL http://CRAN. R-project.org/package=timeSeries. R package version 3022.101.2. [p480]

H. Wickham. ggplot2: Elegant Graphics for Data Analysis. Springer Science \& Business Media, 2009. [p474]

H. Wickham. scales: Scale Functions for Visualization, 2016a. URL http://CRAN. R-project.org/ package=scales. R package version 0.4.0. [p484]

H. Wickham. tidyr: Easily Tidy Data with 'spread()' and 'gather()' Functions, 2016b. URL http: //CRAN. Rproject. org/package=tidyr. R package version 0.4.1. [p484]

H. Wickham and R. Francois. dplyr: A Grammar of Data Manipulation, 2015. URL http://CRAN. Rproject. org/package=dplyr. R package version 0.4.3. [p484]

A. Zeileis and G. Grothendieck. zoo: S3 infrastructure for regular and irregular time series. Journal of Statistical Software, 14(6):1-27, 2005. URL http://www. jstatsoft. org/v14/i06/. [p480]

A. Zeileis, F. Leisch, K. Hornik, and C. Kleiber. strucchange: An R package for testing for structural change in linear regression models. Journal of Statistical Software, 7(2):1-38, 2002. URL http: //www. jstatsoft.org/v07/i02/. [p481]

Yuan Tang

Uptake Technologies, Inc.

600 West Chicago Ave, Chicago, IL 60654

United States

terrytangyuan@gmail.com

Masaaki Horikoshi

Accenture Japan Ltd. 
Akasaka Intercity 1-11-44 Akasaka Minato-ku, Tokyo

Japan

sinhrks@gmail.com

Wenxuan $\mathrm{Li}$

Department of Agricultural Economics, Purdue University 403 W State Street, West Lafayette, IN, 47907

United States

wenxuan. tess@gmail.com 